ÉGYPTE monde arabe

\section{Égypte/Monde arabe}

$3 \mid 2000$

La censure ou comment la contourner

\title{
Une table tombée du ciel
}

Traduction de l'arabe et notes par Ghislaine Alleaume

\section{Youssef Idris}

Traducteur : Ghislaine Alleaume

\section{(2) OpenEdition}

\section{Journals}

Édition électronique

URL : https://journals.openedition.org/ema/815

DOI : 10.4000/ema.815

ISSN : 2090-7273

\section{Éditeur}

CEDEJ - Centre d'études et de documentation économiques juridiques et sociales

\section{Édition imprimée}

Date de publication : 30 juin 2000

Pagination : 229-236

ISBN : 2-87027-856-X

ISSN : 1110-5097

\section{Référence électronique}

Youssef Idris, « Une table tombée du ciel », Égypte/Monde arabe [En ligne], 3 | 2000, mis en ligne le 08 juillet 2008, consulté le 07 juillet 2022. URL : http://journals.openedition.org/ema/815 ; DOI : https:// doi.org/10.4000/ema.815

Ce document a été généré automatiquement le 7 juillet 2022

Tous droits réservés 


\title{
Une table tombée du ciel
}

Traduction de l'arabe et notes par Ghislaine Alleaume

\author{
Youssef Idris
}

Traduction : Ghislaine Alleaume

1 Voir quelqu'un courir dans une rue de Minyat al-Nasr, en voilà un événement ! Rares ici sont ceux qui courent... À quoi bon courir, quand rien au village ne le requiert ?... Les rendez-vous ne s'y comptent pas en minutes et secondes... Les trains, même, y suivent le lent mouvement du soleil, puisqu'il en passe un à l'aube, un à la méridienne et un au crépuscule. Aucun tumulte, ici, ne vient irriter les nerfs ni ne pousse à la précipitation ou à la vitesse. Tout est lent, calme, raisonnable et tout se plaît et se délecte à cette lenteur et à ce calme. La vitesse n'est jamais de mise. La hâte est le fait du diable ! Voir quelqu'un courir dans une rue de Minyat al-Nasr, en voilà un événement! Comme la sirène de police secours, c'est le signal, sans doute aucun, de quelque chose d'excitant. Et quoi de plus beau que de voir arriver dans la bourgade calme et lente quelque chose d'excitant!

2 Ce vendredi-là, il n'y en eut pas qu'un à courir dans Minyat al-Nasr. Ce fut, à la vérité, un mouvement de course général. Et personne n'en savait la raison... Les rues et les ruelles baignaient dans leur calme sempiternel et étaient frappées de cette immobilité qui s'établit d'ordinaire après la prière du vendredi, lorsque le sol est éclaboussé d'eau de lessive mêlée de mousse, de javel et d'odeur de savon bon marché, que les femmes, à l'intérieur, s'affairent à préparer le déjeuner et que les hommes, à l'extérieur, badaudent et baguenaudent, attendant que tout soit prêt. Tout ce calme, soudain, fut rompu par d'énormes et grosses jambes qui couraient, ébranlant les maisons. Le coureur passa devant un groupe assis sur le pas d'une porte, sans oublier, tout en courant, de le saluer à la volée. Les assis lui rendirent son salut et voulurent lui demander ce qui le faisait courir ainsi, mais il était déjà passé. Alors, ils se levèrent, voulurent savoir, et, bien sûr, ne le purent. Alors, la curiosité aidant, ils se mirent en marche. Puis quelqu'un proposa de presser le pas et ils se hâtèrent. Au bout du compte, ils se trouvèrent à courir, et, comme ils n'oubliaient pas de saluer les groupes d'assis, les groupes se levèrent et il ne fallut pas longtemps pour qu'ils se trouvassent à courir eux aussi. 
Car, si mystérieuse que fût la raison, il ne faisait aucun doute qu'à la fin, elle serait connue, comme il ne faisait aucun doute que, sous peu, tous seraient rassemblés sur les lieux de l'événement... Le village est petit. Impossible de s'y perdre ou de s'y cacher, on en fait le tour avant d'être essoufflé. De fait, il ne s'était pas écoulé bien longtemps qu'une forte foule était assemblée sur l'aire à blé. Tous ceux à qui courir était possible étaient déjà arrivés et il ne restait, éparpillés en chemin, que les très vieux et les moins vieux qui avaient préféré marcher à petit pas, pour bien marquer leur âge respectable et tout ce qui les séparait des jeunes gens ou des gamins. Mais ils se hâtaient eux aussi, désireux qu'ils étaient d'arriver avant que les âges ne soient passés et que l'événement ne soit devenu légende.

Minyat al-Nasr, comme tous les villages du vaste monde, avait du vendredi une crainte superstitieuse; tout événement se produisant ce jour-là ne pouvait être qu'une catastrophe certaine. Bien plus, par un excès de pessimisme, les habitants de Minyat alNasr se gardaient bien ce jour-là d'entreprendre la moindre tâche, de crainte que ce ne soit un échec, et, de la sorte, tous les travaux y étaient ajournés au samedi. À qui demandait la raison d'une telle crainte, on répondait que le vendredi porte malheur, parce qu'il compte toujours une heure de déveine. Il semble cependant que la raison en soit tout autre et que cette heure de déveine ne soit qu'un prétexte, un moyen, tout bonnement, pour les paysans, de remettre au samedi le travail du vendredi, lequel, ainsi, devient jour de repos. Mais repos est un mot honni des paysans. Le repos est une offense faite à leur rudesse et leur inlassable capacité à travailler. Le repos, seuls en ont besoin les citadins aux chairs blanches qui travaillent à l'ombre et encore, s'essoufflent! Le repos hebdomadaire est une mode stupide, donc. Toutefois, le vendredi étant un jour qui porte malheur, marqué par la déveine, il est permis, ce jourlà, et ce jour seulement, de remettre au lendemain ce que l'on aurait dû faire le jour même.

5 Ainsi donc, tous s'attendaient à ce que la raison de ce mouvement de course fût quelque grand malheur survenu à quelqu'un. Mais lorsqu'ils arrivèrent sur l'aire à blé, ils ne trouvèrent ni quadrupède occis, ni incendie en cours, ni quelque homme en égorgeant un autre. Ils trouvèrent le cheikh 'Alî, debout au milieu de l'aire, en proie à une violente colère. Il avait enlevé sa gallabeyya et son turban, s'était saisi de son bâton et l'agitait frénétiquement. À qui demandait l'histoire, les premiers arrivés répondaient :

«Voilà que le cheikh menace d'abjurer!»

6 Les gens alors riaient : sans l'ombre d'un doute, c'était là une nouvelle extravagance du cheikh cAlî, lequel était en lui-même une extravagance. Sa tête avait la grosseur de celle d'un âne, ses yeux étaient immenses et ronds comme ceux d'une chouette et il avait au coin de chaque paupière un caillot de sang. Sa voix, lorsqu'il parlait, sortait enrouée et étouffée comme le bruit d'un réchaud qui s'étouffe et charbonne. Il n'avait pas de sourire et, d'ailleurs, ne souriait jamais. Quand il était content - ce qui arrivait rarement -, il s'esclaffait. Quand il n'était pas content, il avait un rictus mauvais. Au moindre mot qui lui déplaisait, son sang virait jusqu'à tourner en mazout et il tombait à bras raccourcis sur celui qui l'avait prononcé. Il pouvait aller jusqu'à l'assommer de son poing aux doigts comme des poteaux ou d'un coup de son bâton - un gourdin d'un bambou énorme, à la pointe ferrée, qu'il aimait, qu'il chérissait même et qu'il appelait "l'Agha des Janissaires »'.

7 Son père, pourtant, l'avait envoyé étudier à al-Azhar ${ }^{2}$. Là, son professeur commit un jour l'erreur de lui dire : «Tu es bête comme une mule. » Et le cheikh cAlî ne trouva rien 
de mieux que de lui répondre: «Et toi, comme soixante mules!» Évidemment, il fut renvoyé et se retrouva à Minyat al-Nasr, prédicateur et imam de la mosquée. Mais, un beau jour, oublieux de ce qu'il faisait, il se prosterna trois fois durant la prière du vendredi. Comme les fidèles, derrière lui, essayaient de l'avertir de son erreur, il les envoya tous au diable et, de ce jour, renonça à l'imamat et à la mosquée. Par leur faute, il renonça même à prier. Il s'enticha alors des cartes et s'adonna au jeu jusqu'à vendre tout ce qu'il possédait, pour jurer ensuite, par la répudiation, de ne plus y toucher. Muhammad effendi, le professeur de l'école primaire du bourg voisin, ouvrait alors, dans le village, une échoppe d'épicier. Il proposa au cheikh de tenir la boutique quelques heures dans la matinée et celui-ci accepta. Mais il n'y travailla que trois jours. Le quatrième jour, Muhammad effendi, debout devant sa boutique, dégoulinait de halva! Car le cheikh 'Alî avait découvert qu'il avait soudé un morceau de fer sous le plateau de la balance.

8 «Mais tu es un voleur !» s'était-il exclamé et Muhammad effendi avait à peine eu le temps de lui dire : «Allons, allons, cheikh cAlî, réfléchis un peu... c'est ton gagne-pain à toi aussi! », que déjà, le cheikh l'avait coiffé d'un bloc de halva!!... Aussi, de ce jour, plus personne ne s'était hasardé à lui proposer du travail. D'ailleurs, si quelqu'un s'y était risqué, le cheikh lui-même n'aurait guère mis d'enthousiasme à accepter.

Ce cheikh cAlî était affreux, grincheux, on ne lui connaissait aucun travail et pourtant personne au village ne le détestait. Tous, au contraire, l'aimaient, l'adoraient et l'on se faisait des uns aux autres le récit de ses extravagances. Il n'était plus délicieux moment que celui où, faisant cercle autour de lui, on s'amusait à le provoquer pour déclencher sa colère. Sa colère faisait rire. Quand il s'emportait, ses traits se gâtaient, sa voix s'étouffait.... Impossible d'y résister, c'était à mourir de rire! Et, eux l'excitant sans cesse, lui s'emportant sans cesse, l'on restait à rire jusqu'à ce que, peu à peu, l'assemblée se disperse. "Quel numéro, ce cheikh cAlî!", s'exclamait-on de toutes parts. Et on le laissait seul verser le trop-plein de sa colère sur Abû Ahmad. Abû Ahmad était le nom qu'il donnait à sa pauvreté, désignant ainsi son seul ennemi mortel. Il en parlait comme d'un être réel, ayant un nom et fait de chair et de sang. Les séances commençaient lorsque quelqu'un demandait :

«Eh, dis-donc, cheikh 'Alî, que t'a fait Abû Ahmad aujourd'hui ?»

10 Pour le coup, le cheikh se mettait dans une véritable rogne. Car il n'aimait pas que l'on parlât de sa pauvreté. Qu'il en parle, lui, soit. Mais que les autres seulement l'évoquent, c'en était trop, cela le mettait hors de lui. Car le cheikh cAlî était, en réalité, extrêmement pudique, en dépit de la rudesse de ses traits et de son langage. Il préférait rester des jours sans tabac, plutôt que de demander à quelqu'un de lui rouler une cigarette. Il avait en permanence sur lui une aiguille et du fil pour repriser sa gallabeyya lorsqu'elle se déchirait d'usure. Et lorsqu'elle était sale, il s'éloignait du village, lavait ses vêtements et restait nu jusqu'à ce qu'ils soient secs. Aussi son unique turban était-il le plus propre du village.

11 Rien d'étonnant donc, à ce que les villageois de Minyat al-Nasr aient ri de cette nouvelle extravagance. Les rires, pourtant, mouraient aussitôt et les langues rentraient dans les gosiers, comme piquées par des scorpions. Car abjurer est un mot abhorré. Le village, comme tous les villages, vivait dans la paix du Seigneur. On y trouvait, tout ce que l'on trouve partout ailleurs, des hommes de bien qui ne connaissent que leur travail et leur maison; des petits voleurs qui chapardent les épis de maïs et des grands qui rôdent dans les enclos et emmènent le bétail par le museau; des commerçants qui comptent en 
livres et des qui comptent en piastres; des femmes légères à l'insu de tous et d'autres qui le sont au vu et au su de tout un chacun; des qui disent vrai, des qui mentent et des gardes champêtres ; des malades, des vieilles filles, des honnêtes gens, bref, tout ce que l'on trouve partout ailleurs. Et tous, on les voyait à la mosquée lorsque le muezzin lançait l'appel à la prière et il ne s'en trouvait pas un qui ne jeûnât en Ramadan. Il y avait des règles, que l'on observait et qui réglaient la vie de chacun. C'était les " principes » : le voleur ne s'en prend pas au voleur ; nul métier n'est à mépriser; on ne va pas à l'encontre du sentiment général... Et voilà que le cheikh cAlî était planté là et s'adressait au ciel sans tambour ni trompette!

Ils riaient un peu, mais avaient à peine entendu ce qu'il disait, que l'angoisse les poignait. Le cheikh était tête nue et sa courte chevelure brillait de sueur et de graisse. L'« Agha des Janissaires » dans la main droite, les yeux lançant des éclairs, le visage empourpré de fureur, la face tournée vers le ciel, il disait :

«Mais qu'est-ce que tu attends de moi? Tu peux me dire ce que tu attends de moi? Al-Azhar... je l'ai quitté, je laisse ça à cette poignée d'enturbannés qui se prennent pour les garants de la piété... Ma femme... je l'ai répudiée... La maison... je l'ai vendue... Et c'est sur moi que tu fais tomber Abû Ahmad, et sur moi seul !... Il n'y a donc que moi dans ce bas monde? Pourquoi, bon Dieu, ta colère tombe pas un peu sur Churchill ou Zenhower'? Il n'y a que moi ?... Tu ne peux pas t'en prendre à quelqu'un d'autre? Mais qu'est-ce que tu attends de moi, cette fois-ci, hein, quoi ? Les autres fois, tu m'affamais un jour... je supportais. Je me disais, mon garçon, c'est comme si on était en Ramadan... c'est l'affaire d'un jour... ça va passer. Mais cette fois, je n'ai rien mangé depuis avant-hier soir. Et les cigarettes... voilà une semaine que je suis sans cigarettes... Et une pipe, bon Dieu de bon Dieu, ça fait bien dix jours que j'en ai même pas senti l'odeur! Et tu racontes qu'au Paradis il y a du miel, des fruits, des fleuves de lait... Pourquoi tu ne m'en donnes pas alors? Tu attends que je crève de faim pour m'envoyer là-haut me nourrir de tes bienfaits? Non, mon beau monsieur ! Je ne marche pas ! Tu me fais vivre aujourd'hui... après, ça m'est égal, tu m'enverras où tu voudras. Allez, vieux frère, éloigne un peu de moi cet Abû Ahmadlà. Envoie-le donc en Amérique ! Il a fallu que ça tombe sur moi ! Mais pourquoi tu me tortures comme ça? regarde-moi.... Je n'ai que cette gallabeyya et l'« Agha des Janissaires ". Qu'est-ce que tu veux donc? Bon, ou tu me donnes à manger, tout de suite... ou tu me rappelles à toi sur le champ. Tu vas me donner à manger, oui ou non?»

13 Le cheikh 'Alî était dans une surexcitation telle que l'écume lui en venait aux lèvres. Il était inondé de sueur et sa voix débordait d'une irrépressible hargne. Les gens de Minyat al-Nasr se tenaient là, le cœur prêt à rompre d'effroi. Ils craignaient que le cheikh n'allât vraiment jusqu'à abjurer. Mais ils avaient plus encore à redouter. Car les propos qu'il tenait étaient en eux-mêmes un péril. Dieu Très-Haut pouvait s'en offenser et leur village avoir à subir de ce fait des représailles qui s'abattraient sur les récoltes et sur les champs. Par son langage, le cheikh cAlî était une menace pour la paisible communauté tout entière. Il fallait à tout prix le faire taire.

14 Aussi des malins commencèrent-ils à proférer de loin quelques paroles d'apaisement, exhortant le cheikh à reprendre ses esprits et à se taire. Délaissant le ciel un instant, le cheik 'Alî se tourna vers eux :

«Me taire, bande de culs-terreux! et pourquoi donc? Me taire, jusqu'à quand? Jusqu'à ce que je sois mort de faim? Vous tremblez, hein, pour vos maisons, vos femmes, vos champs... ? Eh bien, tremblez! Moi je n'ai rien à perdre! Si je l'irrite, celui-là, qu'il vienne me prendre! Mais sur ma foi, je jure bien que si quelqu'un vient me chercher, même si c'est Azraël', je lui pulvérise l'« Agha des Janissaires » sur la tête ! Bon Dieu, je ne me tairai que s'il m'envoie une Table Servie', et tout de 
suite ! Je ne vaux pas moins que Marie... c'était jamais qu'une femme après tout, et moi je suis un homme! Et puis, elle n'était pas pauvre, elle! Moi, j'en ai ma claque d'Abû Ahmad! Sur ma foi, je ne me tairai que s'il m'envoie tout de suite une Table Servie.»

Se tournant alors vers le ciel, il reprit :

«Tu entends? Ou tu l'envoies tout de suite, sur le champ, ou alors, je ne me retiens plus, je lâche tout! Tu n'as qu'à faire ce qu'on te demande. Une table, tout de suite, avec une paire de poulets, un plat de miel et quelques pains chauds... attention, hein, bien chauds les pains! Et n'oublie pas la salade! Bon Dieu, je compte jusqu'à dix et si tu n'as pas envoyé la table, on verra ce qu'on verra!»

Le cheikh se mit à compter. Les cœurs de Minyat al-Nasr comptaient avec lui, en le devançant, et les nerfs commençaient à craquer. Il fallait absolument faire quelque chose pour mettre un terme à cette situation. Quelqu'un proposa que quelques costauds $\mathrm{du}$ village l'encerclent, lui tombent dessus, lui ferment la bouche et lui flanquent une raclée dont il se souviendrait. Mais un seul regard jeté par le cheikh cAlî de ses yeux enflammés de rage démente suffit à annihiler la suggestion. Car de toute évidence, il était impossible de maîtriser le cheikh sans qu'il n'ait le temps, au préalable, d'asséner un ou deux coups de son "Agha des Janissaires ». Et chacun pressentait que le coup pouvait bien être son lot. Et puis, un homme capable de menacer de pulvériser la tête d'Azraël pouvait bien en faire autant à n'importe lequel d'entre eux ! Il se trouva alors une tête de linotte pour dire :

«Mais enfin, toute ta vie, tu as eu faim, bonhomme, qu'est-ce qui te prend aujourd'hui?»

Il eut en retour un regard de braise du cheikh, qui lui répondit :

«Cette fois-ci, cAbd al-Gawâd, vieux citron, l'histoire a assez duré. »

Un autre lui lança :

"Mais quand tu as faim, mon vieux, pourquoi tu ne nous le dis pas? On te donnerait à manger. Ça vaudrait mieux que ce discours insensé que tu nous tiens là!»

19 Le cheikh hurla :

«Que je vous demande quoi que ce soit, moi! Que je mendie auprès de vous, bande de ventres-creux! Vous êtes plus affamés que moi. Et j'irais mendier auprès de vous! Non, mon beau, c'est à lui, là-haut, que je m'adresse. Et s'il ne me donne rien, je sais ce qui me reste à faire! »

‘Abd al-Gawâd lui répondit :

« Aussi, si tu travaillais, tu mangerais à ta faim ! Tu n'as pas honte !»

21 À ces mots, la colère du cheikh fut à son comble. Il verdit, se mit à trembler et, partageant son discours entre la foule et le ciel, il vociféra :

"Qu'est-ce que ça peut te foutre, 'Abd al-Gawâd, fils de Sett Abûhâ'! Non, je ne travaille pas. Je ne veux pas travailler. Je ne sais pas travailler. Je ne trouve pas de travail. Vous croyez que c'est un travail que le vôtre, bande de bouseux! Un travail d'ânes, oui ! Et moi, je ne suis pas un âne! Moi, je ne peux pas m'échiner toute la journée comme ça... rester accroché à un champ comme une bête de somme. Des bêtes, voilà ce que vous êtes! Allez au diable, tous autant que vous êtes! Moi, je ne travaillerai pas. Et par le Prophète, même si je dois crever de faim, je ne ferai jamais ce que vous faites!»

22 Sa colère était telle que, malgré eux, et en dépit de l'horreur de la situation, ils commençaient à en rire. Le cheikh cAlî eut un énorme soubresaut et reprit : 
«Ah, c'est comme ça! Je compte jusqu'à dix et, par le Prophète, si tu ne m'envoies pas une Table Servie, j'abjure et je commets l'irréparable! »

Il était clair que le cheikh disait vrai et ne se rétracterait pas. Il avait décidé de dépasser les bornes et toute l'affaire ne pouvait qu'avoir d'insurmontables conséquences. Il commença à compter. Des gouttes de sueur perlaient sur tous les fronts. La chaleur de l'après-midi se fit insupportable. Au point même que certains murmurèrent que la vengeance du Seigneur était en train de s'accomplir et que cette horrible chaleur n'était qu'un prélude au formidable incendie qui allait se déchaîner, emportant le blé sur pied comme les récoltes rentrées. Quelqu'un commit l'erreur de dire :

« Allez donc lui chercher un bout de pain, ça le calmera peut-être !»

La phrase, sans doute, parvint jusqu'aux oreilles du cheikh, en dépit de ce qu'il comptait d'une voix forte et haute, car il se tourna vers la foule et hurla :

«Un bout de pain, bande de va-nu-pieds! un bout de votre pain fétide et de votre fromage bouffé par les vers! Vous croyez que je vais avaler ça! ? Bon Dieu, je ne me tairai que s'il me fait descendre, là... une Table Servie, avec une paire de poulets. »

Il y eut des murmures dans la foule et une brave femme qui se trouvait là, proposa :

"J'ai fait un beau plat de cornes grecques, pépère. Tu veux que je t'en apporte une assiette?»

Le cheikh hurla :

«La ferme, femme! Des cornes grecques! Encornés vous-mêmes! Même vos cervelles sont en cornes grecques! Tout le village pue la corne grecque avariée ! » - «Chez nous, il y a du poisson frais de ce matin », proposa Abû Sarhân, « on vient de l'acheter à Ahmad le pêcheur. Si ça te dit... »

- «Tu parles d'un poisson», rugit le cheikh, "gros comme un pois chiche! Village de crève-la-faim ! Non, bon Dieu, s'il ne m'envoie pas une paire de poulets et tout ce que j'ai demandé, je m'en vais blasphémer et il arrivera ce qui arrivera! »

La situation était devenue insupportable. Ou l'on se taisait et le village courait à sa perte, ou l'on faisait taire le cheikh, coûte que coûte. Cent gosiers lancèrent cent invitations à déjeuner, que cent fois, il refusa, disant :

«Moi, je suis là à danser devant le buffet... ça fait trois jours que personne ne m'a donné un bout de pain et maintenant c'est à qui m'invitera! Eh bien, non! Je ne me tairai que lorsqu'il m'enverra la Table Servie !»

Il y eut des mouvements de tête interrogateurs... Qui a cuisiné aujourd'hui ? car tout le monde ne cuisine pas chaque jour et, pour les villageois, avoir un bout de gras ou un poulet est un événement grandiose. Finalement, on trouva chez 'Abd al-Rahmân une pièce de veau bouilli, intacte, d'une livre, que l'on apporta sur une table basse, avec des radis, quatre galettes de pain et une tête d'oignon.

"Ça te va, ça?»

Le regard du cheikh allait du ciel à la table. À chaque regard jeté au premier, ses yeux lançaient des éclairs. À chaque regard jeté à la seconde, son visage s'empourprait de colère. La foule gardait un profond silence. À la fin, le cheikh laissa tomber ces mots :

«Il reste, village de va-nu-pieds, que je demande une Table Servie et que vous m'apportez une table basse ! Et les cigarettes, où sont-elles?» Quelqu'un offrit son paquet de tabac. Le cheikh tendit la main et prit un gros morceau de viande. Mais avant de l'engloutir, il dit encore : «Et la boulette de noir "?»

- «Tout ce que tu veux, mais pas ça!»

- «Ah, c'est comme ça!», reprit-il, furieux. 
délaissant la nourriture, il enleva gallabeyya et turban et se mit à agiter son bâton, menaçant à nouveau d'abjurer. Il ne se tut que lorsqu'on lui eut amené Mandûr, le marchand d'opium, qui lui en coupa un bout qu'il roula en boulette.

«Tiens, mon vieux, prends donc, tu ne l'as pas volé! On se conduit comme des brutes... on ne savait pas que ça te gênait de demander. On passe des heures ensemble, on se régale de toi et puis, après, on fait la sourde oreille et on s'en va, te laissant seul. Pourtant c'est à nous de prendre soin de toi. Car sans toi et ton Abû Ahmad, que vaudrait notre village, hein... pas grand-chose! Écoute, tu continues à nous faire rire et, nous, on te donne à manger, hein, qu'est-ce que tu en dis ?»

Ce fut un nouvel accès de rage. Le cheikh vola derrière Mandûr, au comble de la fureur, brandissant « l'Agha des Janissaires », dont il faillit bien lui asséner un coup.

«Ah, je vous fais rire! Tu me prends pour un pitre, espèce de savate! Que la malédiction du Ciel s'abatte sur toi et sur les tiens!»

Mandûr courait devant lui en riant et c'est en riant aussi que les autres assistaient à la poursuite. Ils rirent encore lorsque le cheikh se lança à leur trousse en les accablant d'injures et de malédictions.

Le cheikh 'Alî vit toujours à Minyat al-Nasr, commettant chaque jour quelque excentricité et toujours prompt à la colère. Et ses colères font toujours rire les villageois, à ceci près que, depuis ce jour-là, ils savent à quoi s'en tenir. Sitôt qu'ils le voient, debout au milieu de l'aire à blé, ayant enlevé gallabeyya et turban, tenant à la main son "Agha des Janissaires ", qu'il agite en direction du ciel, ils comprennent qu'une fois encore, ils l'ont oublié et ont laissé Abû Ahmad prendre sur lui trop d'emprise. Alors, avant même que ne sorte de sa bouche le moindre mot de blasphème, la table est là, avec tout ce qu'il désire. Et il veut bien, parfois, se contenter de ce que Dieu lui envoie!

\section{NOTES}

1.Littéralement le Katkhuda, titre donné à l'Agha des Janissaires, chargé de la police urbaine.

2.La grande mosquée du Caire.

3.Eisenhower.

4.L'ange de la mort chez les musulmans.

5.Allusion à la cinquième sourate du Coran (al-Mahdiyya). Il s'agit de la Table Servie demandée par les apôtres à Jésus en signe de la toute-puissance divine.

6.Littéralement, fils d'une femme qui porte la culotte dans son ménage, une manière de dire qu'il ne vaut pas grand chose.

7.Euphémisme pour désigner l’opium. 